\title{
Avaliação nutricional e prevalência de doenças crônicas não transmissíveis em idosos pertencentes a um programa assistencial
}

\author{
Nutritional evaluation and prevalence of not transmissible \\ chronic disease in elderly participating in an assistance program
}

\author{
Júlia M acedo Bueno ${ }^{1}$ \\ Hercia Stampini Duarte M artino ${ }^{2}$ \\ M aria Fernada Scareli Fernandes ${ }^{1}$ \\ Luciana Silva Costa ${ }^{1}$ \\ Roberta Ribeiro Silva ${ }^{1}$
}

${ }^{1}$ Departamento de N utrição, UNIFAL-MG. RuaGabriel M onteiro da Silva 714, Centro. 37130-000 Alfenas MG.

betaribeiro@hotmail.com ${ }^{2}$ Departamento deNutrição eSaúde, Universidade Federal deViçosa.
Abstract The purpose of this study was to evaluate the nutritional state and the prevalence of not transmissible chronic disease in elderly, who were participating in an assistance program of the Federal University of Alfenas (UNIFAL-MG), Alfenas, $M$ inas Gerais, Brazil. Socio-economical, anthropometric and biochemical variables, as well as systemic blood pressure were collected from 82 individuals between 60 and 87 years of age, 90,2\% of them female. According to the body mass index (BMI) $52,4 \%$ of the studied sample were overweight, $28,0 \%$ eutrophic and $19,5 \%$ underweight; $37,8 \%$ presented high body fat percentage (BF\%). With regard to the relation $\mathrm{BM} \mathrm{I} / \mathrm{BF} \%, 63,4 \%$ of theelderly with overweight, $12,5 \%$ of theeutrophic and $11,8 \%$ of the underweight presented high $\mathrm{BF} \%$. The waist-to-hip ratio revealed $40,2 \%$ at high risk and $12,2 \%$ at very high risk of developing cardiovascular disease. In addition, $22,0 \%$ had high blood pressure. Thebiochemical tests revealed that $39,3 \%, 39,3 \%$ and 3,3\% presented higher plasma cholesterol, triglyceride and glucose levels re spectively. There is a need for continuous nutritional education programs and monitoring of the nutritional and health status for improving the quality of life of the studied individuals. Key words Nutritional evaluation, Elderly, Hypertension, $\mathrm{N}$ ot transmissible chronic diseases
Resumo Objetivou-se avaliar o estado nutricional ea prevalência de doenças crônicas não transmissíveis em idosos que participavam de um programa assistencial da U niversidade Federal deAIfenas (U N IFAL-M G), Alfenas, M G, Brasil. Foram coletadas variáveis socioeconômicas, antropomé tricas, bioquímicas e pressão arterial sistêmica de 82 indivíduos de 60 a 87 anos, sendo $90,2 \%$ do sexo feminino. Encontrou-se 52,4\% desobrepeso, 28,0\% deeutrofia e $19,5 \%$ de baixo peso pelo I M C e37,8\% apresentaram percentual de gordura corpórea (\%GC) elevada. Relacionando-seĺndicedeM assa Corpórea (IM C) com \% GC, 63,4\% dosidosos com sobrepeso, $12,5 \%$ dos eutróficos e $11,8 \%$ dos com baixo peso apresentavam \%GC elevado. A razão da circunferência da cintura e do quadril (RCQ) revelou $40,2 \%$ em risco al to e $12,2 \%$ em risco muito alto para o desenvolvimento de doenças cardiovasculares. Quanto à pressão arterial, 22,0\% eram hipertensos. Em relação aos exames bioquímicos, observou-se que $39,3 \%$, 39,3\% e 3,3\% dos idosos apresentavam valores plasmáti cos el evados de colesterol, triglicerídeos e glicose, respectivamente. Programas de educação nutricional continuada e de monitoramento do estado nutricional e de saúde são necessários para melhoria da qualidade de vida destes indivíduos estudados.

Palavras-chave Avaliação nutricional, Idosos, D oenças crônicas não transmissíveis 
Introdução

De acordo com o censo de 2000, o número de idosos no Brasil era de 14.546 .029 pessoas, representando um aumento de 35,6\% em relação ao ano de 1991. As estimativas apontam para a possibilidade de, nos próximos vinte anos, no Brasil, o número de idosos ultrapassar $30 \mathrm{mi}-$ Ihões de pessoas, devendo representar quase $13,0 \%$ da população ${ }^{1}$.

Com o processo de envelhecimento populacional, aumenta cada vez mais a necessidade do estudo dos fatores que incidem sobre a prevalência das doenças crônicas não transmissíveis associadas à idade, bem como 0 aprofundamento da compreensão sobre o papel da nutrição na promoção emanutenção da independência eautonomia dos idosos?

As alterações relacionadas à idade ocorrem praticamente em todas as partes do corpo, trazendo diversas mudanças funcionais ao organismo idoso. Dentre elas, a redução da massa magra, aumento do tecido adiposo corpóreo e a menor eficiência de bombeamento do coração, podendo haver diminuição do fluxo sangüíneo. Também, o olfato e o paladar podem tornar-se menos agudos, a mastigação se tornando difícil devido à perda dos dentes, a menor secreção de ácido clorídrico e bile dificultando a digestão e a perda do tônus do trato gastrointestinal levando à constipação ${ }^{3}$.

Assim, há um aumento do risco de desenvolver desnutrição, já quea deficiência nutricional é um problema relevantena população idosa, pois com o avanço da idade, os gerontes apresentam condições peculiares, devido a alterações do próprio envel hecimento, doenças sistêmicas e/ou situação socioeconômica, que condicionam o seu estado nutricional ${ }^{4}$.

Por outro lado, a obesidade leva aos distúrbios das condições de saúde do organismo, sendo representados por distúrbios psicológicos, sociais, aumento do risco de morte prematura e 0 aumento derisco de doenças de grandemorbimortalidade, como diabetes mellitus, hipertensão arterial, hiperlipidemias, doenças cardiovasculares e câncer. Além disso, pode estar associada a outras doenças que podem interferir na qualidade de vida do indivíduo obeso 5 .

A nutrição e a alimentação na terceira idade ainda são pouco exploradas, não tendo recebido a devida atenção ${ }^{6}$. Com 0 aumento de pessoas acima dos 60 anos de idade, aumenta a necessidade de estudos queinvestiguem o perfil nutricional e o estado de saúde para que as propostas de educação continuada tenham adesão e impacto na qualidade de vida desses indivíduos. Dentro desse contexto, o presente estudo teve como objetivo avaliar o estado nutricional e a prevalência de doenças crônicas não transmissíveis em idosos regularmente inscritos em um programa assistencial da Universidade Federal de Alfenas (UNIFAL-MG).

\section{M etodologia}

População estudada

0 estudo, do tipo transversal, foi realizado com 82 idosos, de ambos os sexos, com 60 anos ou mais, regularmente matriculados na Universidade Aberta à Terceira I dade (UNATI) da U NIFAL-M G, em Alfenas, M G. A coleta de dados foi realizada no período defevereiro a julho de2005, pelas pesquisadoras devidamente treinadas.

A UNATI contava com 242 inscritos, sendo quesomente $206,0(85,1 \%)$ freqüentavam as atividades do programa. Destes, $92(44,7 \%)$ apresentavam idade igual ou superior a 60 anos e foram convidados a participar do estudo. Desses indivíduos, 10,9\% recusaram-se a participar da pesquisa. Quanto à realização dos exames de glicemia, colesterol e triglicerídeos, somente 61 $(74,4 \%)$ dos 82 indivíduos aceitaram submeterse aos testes.

O presente trabalho foi aprovado pelo Comitê de Ética em Pesquisa da U NIFAL-M G, sendo realizado posteriormente à assinatura de um termo de consentimento esclarecido para todos os participantes.

\section{Coleta dos dados}

Para caracterização da população em estudo, aplicou-se um questionário socioeconômico. $\mathrm{Na}$ determinação do diagnóstico do estado nutricional, a partir da antropometria, as medidas recomendadas ${ }^{7}$ e aferidas foram: peso, estatura, circunferência da cintura e do quadril.

\section{Avaliação antropométrica}

Para mensuração do peso, utilizou-se balança eletrônica digital portátil, tipo plataforma, marca Kratos, com capacidade para $150 \mathrm{Kg}$ e sensibilidade de 50g. A estatura foi aferida utilizando o antropômetro Alturaexata, de altura máxima de 2,13m e precisão de $1 \mathrm{~mm}$, conforme metodologia prescrita por Jelliffe 8 . O Índice de M assa 
Corporal (IMC) foi calculado através da relação entre peso corporal total, em quilogramas, e estatura, em metros ao quadrado. A avaliação do estado nutricional foi realizada por meio do IMC, utilizando-se os pontos de corte para idosos propostos pelo N utrition Screening I niciative?.

As circunferências da cintura e do quadril foram mensuradas utilizando fita métrica inelástica, com extensão de $2 \mathrm{~m}$, flexível einelástica, dividida em centímetros e subdivida em milímetros. A circunferência da cintura (CC) foi obtida durante a expiração normal, sendo circundada a menor circunferência horizontal localizada abaixo das costelas eacima da cicatriz umbilical. A do quadril (CQ) foi verificada na região glútea, sendo circundada a maior circunferência horizontal entre a cintura e os joelhos. Os dados obtidos pela relação cintura-quadril (RCQ), que mede o risco para o desenvol vimento de doenças cardiovasculares, foram comparados com referenciais propostos por Bray e Gray ${ }^{10}$.

A porcentagem de gordura corporal (\%GC) foi avaliada utilizando o aparelho de bioimpedância bicompartimental da marca Biodynamics BIA 310e. A classificação foi realizada de acordo com os pontos de corte para \%GC adaptados para idosos ${ }^{11}$.

\section{Aferição da pressão arterial}

A aferição da pressão arterial sistólica (PAS) e diastólica (PAD) foi realizada em esfigmomanômetro manual da marca Sankey. A classificação da pressão arterial foi realizada segundo Lip e Chung ${ }^{12}$.

\section{Exames bioquímicos}

A glicemia, colesterol e triglicerídeos foram analisados pelo aparelho da marca Accutrendâ Roche, que utiliza os pontos de corte para os níveis denormal idade da glicose, colesterol etriglicerídeos recomendados pela Sociedade Brasileira de Cardiologia ${ }^{13}$.

\section{Análise estatística}

Os dados obtidos foram armazenados em banco de dados criado no programa Epidata versão 2.0, sendo posteriormente processados eanalisados no programa Epi Info versão $6.0^{14} \mathrm{e}$ Statistical Packagefor the Social Sciencefor Windows(SPSS), versão 10.0, ano 200015. Os dados contínuos foram analisados pelos testes de média, desvio-padrão e correlação de Pearson. Os da- dos categóricos foram analisados pelo teste de qui-quadrado ou teste exato de Fischer. Adotouse o nível de $5 \%$ designificância $(p<0,05)$.

\section{Resultados}

A população em estudo foi composta por 82 indivíduos de 60 a 87 anos, pertencentes ao ProgramaUNATI da UNIFAL-M G, dos quais $90,2 \%$ eram do sexo feminino e 9,8\%, do sexo masculino. A distribuiç̧ão entre as faixas etárias apresentou $73,2 \%$ dos indivíduos entre 60 e 69 anos; $19,5 \%$ entre 70 e 79 anos e 7,3\% com 80 anos ou mais (Tabela 1).

Tabela 1. Caracterização dos idosos pertencentes à UNATI-UNIFAL-M G, Alfenas - M G, 2005.

\begin{tabular}{lrr}
\hline \multicolumn{1}{c}{ Variável } & $\mathrm{n}$ & $\%$ \\
\hline I dade & & \\
60 a 69 anos & 60 & 73,2 \\
70 a 79 anos & 16 & 19,5 \\
80 anos ou mais & 6 & 7,3 \\
Nível de escolaridade & & \\
Ensino fundamental incompleto & 15 & 18,3 \\
Ensino fundamental completo & 14 & 17,0 \\
Ensino médio incompleto & 5 & 6,1 \\
Ensino médio completo & 20 & 24,4 \\
Ensino superior incompleto & 8 & 9,7 \\
Ensino superior completo & 20 & 24,4 \\
Renda familiar mensal & & \\
1 a 2 salários mínimos & 6 & 7,3 \\
3 a 4 salários mínimos & 18 & 21,9 \\
5 a 7 salários mínimos & 34 & 41,5 \\
8 a 10 salários mínimos & 12 & 14,6 \\
> 10 salários mínimos & 12 & 14,6 \\
Estado civil & & \\
Solteiro & 10 & 12,2 \\
Viúvo & 29 & 35,4 \\
Casado & 36 & 43,9 \\
Divorciado & 7 & 8,5 \\
Alterações fisiológicas & & \\
Sensação de plenitude pós-prandial & 30 & 36,6 \\
Intestino preso & 25 & 30,5 \\
Redução de apetite & 24 & 29,3 \\
Alterações no olfato e/ou paladar & 16 & 19,5 \\
Dor ao mastigar & 4 & 4,9 \\
Perda dos dentes & 39 & 47,5 \\
Total & 82 & 100,0 \\
\hline & & \\
\hline & &
\end{tabular}


Quanto ao nível de escolaridade, $24,4 \%$ dos idosos completaram o ensino médio e $24,4 \%$, 0 ensino superior. Em relação à renda, $41,5 \%$ apresentavam renda familiar mensal mínima de cinco a sete salários mínimos, seguida de 21,9\% com três a quatro salários. Em 46,3 e 43,9\% dos casos, uma a duas pessoas e três a quatro eram dependentes dessa renda, respectivamente. Verificou-se, ainda, que $43,9 \%$ dos gerontes eram casados e $35,4 \%$ viúvos (Tabela 1 ).

Na população em estudo, foram avaliadas as alterações fisiológicas decorrentes do envelhecimento. A perda dos dentes esensação de plenitude pós- prandial foram as condições mais relatadas, 47,5 e $36,6 \%$, respectivamente, seguidas de $30,5 \%$ de relato de intestino preso, $29,3 \%$ de redução do apetite, $19,5 \%$ de alterações negativas no olfato e/ou paladar e 4,9\% de dor ao mastigar (Tabela 1).

Observou-se que, $67,1 \%$ dos idosos faziam uso crônico demedicamentos; desses, $42,9 \%$ usavam somente um tipo, $21,4 \%$, dois, $21,4 \%$, trêse $14,3 \%$, quatro tipos. Das drogas utilizadas, $46,3 \%$ eram anti-hipertensivas, 4,9\%, hipocolesterolemiantes e $4,9 \%$, hipoglicemiantes.

Os valores médios dos dados contínuos dos indivíduos estudados estão apresentados na Tabela 2 .

Segundo dados do Índice de M assa Corpórea (IMC), a maior prevalência foi de sobrepeso $(52,4 \%)$, seguido de eutrofia ( $28 \%$ ) e de baixo peso (19,5\%). Entretanto, $62,2 \%$ dos indivíduos apresentavam Percentual de Gordura Corporal
(\%GC) normal (Tabela 3). Quando foi realizada a análise combinada do estado nutricional, segundo o IMC e o \%GC, observou-se que $63,4 \%$ dos idosos com sobrepeso, $12,5 \%$ dos eutróficos e $11,8 \%$ dos com baixo peso pelo IM C possuíam $\% G C$ elevado $(p=0,0000)$.

A análise da RCQ demonstrou $40,2 \%$ da população em risco alto, 34,2\% em risco moderado, $13,4 \%$ em risco baixo e $12,2 \%$ em risco muito alto para o desenvolvimento de doenças cardiovasculares (Tabela 3).

A proximadamente $30 \%$ dos idosos apresentaram pressão arterial ideal, $24,4 \%$, pressão arterial normal, $24,4 \%$, normal elevada e $22,0 \%$, algum grau de hipertensão arterial. Observou-se que $54,5 \%$ dos idosos eutróficos, $45,5 \%$ dos que apresentavam sobrepeso e 37,5\% dos indivíduos com baixo peso apresentavam algum grau de hipertensão arterial, com valores pressóricos que caracterizavam o quadro de hipertensão arterial sistêmica grau I, II ou hipertensão arterial diastólica isolada.

$\mathrm{Na}$ classificação da RCQ com a pressão arterial, dos idosos que apresentaram pressão arterial normal, $32,8 \%$ tinha risco muito al to e $35,9 \%$, risco moderado, para o desenvolvimento de doenças cardiovasculares. Para os idosos com hipertensão, 66,7\% deles apresentaram RCQ de alto risco e $27,7 \%$, de risco moderado. Analisando-se RCQ com $\%$ GC, foi observado que $35,5 \%$ e $37,4 \%$ dos gerontes com risco alto e com risco moderado, respectivamente, apresentaram \%GC elevado.

Tabela 2. M édia, desvio-padrão e mediana das variáveis contínuas dos idosos pertencentes à UNATIUNIFAL-M G, Alfenas - M G, 2005.

\begin{tabular}{|c|c|c|}
\hline Variável & M édia (_ DP) & Mediana (p25 - p50) \\
\hline Idade (anos) & $66,82+7,1$ & $66,0(60,0-70,0)$ \\
\hline Peso (Kg) & $66,6 \pm 12,1$ & $66,3(59,4-74,3)$ \\
\hline Altura $(\mathrm{cm})$ & $156,4 \pm 5,8$ & $155,2(152,9-159,8)$ \\
\hline IMC $\left(\mathrm{Kg} / \mathrm{m}^{2}\right)$ & $27,3 \pm 5,0$ & $27,1(24,1-30,4)$ \\
\hline Gordura corporal (\%) & $33,7 \pm 7,1$ & $34,6(27,7-38,9)$ \\
\hline Circunferência da cintura (cm) & $90,2 \pm 12,9$ & $90,5(82,0-98,0)$ \\
\hline Razão cintura/quadril (RCQ) & $0,84 \pm 0,06$ & $0,85(0,81-0,9)$ \\
\hline Triglicerídeos plasmáticos (mg/dL) & $251,1 \pm 131,0$ & $191,0(169,0-300,0)$ \\
\hline Colesterol plasmático (mg/dL) & $196,9 \pm 24,9$ & $197,0(181,0-209,0)$ \\
\hline Glicose plasmática (mg/dL) & $93,8 \pm 24,6$ & $90,0(79,0-97,0)$ \\
\hline Pressão arterial sistólica (mmH g) & $127,4 \pm 15,9$ & $125(120,0-140,0)$ \\
\hline Pressão arterial diastólica (mmH g) & $83,838 \pm 12,5$ & $80(80,0-90,0)$ \\
\hline
\end{tabular}


Dos 61 idosos que realizaram os exames bioquímicos, 96,7\% tinham glicemia normal, 59,0 e $50,8 \%$, colesterol e triglicerídeos limítrofe, respectivamente, e ainda, 39,4\% dos idosos apresentaram valores altos para colesterol e triglice rídeos.

Quando foram comparados os níveis de colesterol, glicose e triglicerídeos plasmáticos com o estado nutricional, observou-se glicemia normal em 31 (52,5\%) dosindivíduos com sobrepeso. Os idosos com valores séricos de colesterol limítrofe (50\%) e alto $(58,3 \%)$ estavam com sobrepeso. E aqueles com níveis el evados de colesterol $(58,3 \%)$ e triglicerídeos $(66,7 \%)$ apresentaram sobrepeso. Entretanto, os resultados não apresentaram diferenças significativas (Tabela 4).

Quanto às associações entre as variáveis estudadas, apenas $0 \% \mathrm{GC}$ e o IMC apresentaram associação, com 0 dds de 13,5 e interval o de confiança de 4,0 a 44,8 e $p=0,000$.

Tabela 3. Classificação do índice de massa corporal (IMC), percentual de gordura corpórea $(\% G C)$, relação cintura/quadril (RCQ) e exames bioquímicos dos idosos pertencentes à UNATIUNIFAL-M G, Alfenas - MG, 2005.

\begin{tabular}{llrr}
\hline \multirow{2}{*}{$\begin{array}{c}\text { Variáveis e } \\
\text { índices }\end{array}$} & Classificação & \multicolumn{2}{c}{ Prevalência } \\
\cline { 2 - 4 } & & \multicolumn{1}{c}{$\mathrm{n}$} & $\%$ \\
\hline IMC & Baixo peso & 16 & 19,5 \\
& Eutrofia & 23 & 28,0 \\
& Sobrepeso & 43 & 52,4 \\
\%GC & Total & 82 & 100 \\
& Normal & 51 & 62,2 \\
RCQ & Elevada & 31 & 37,8 \\
& Total & 82 & 100 \\
& Risco baixo & 11 & 13,4 \\
& Risco moderado & 28 & 34,2 \\
& Risco alto & 33 & 40,2 \\
Glicose & Risco muito alto & 10 & 12,2 \\
& Total & 82 & 100 \\
& Normal & 59 & 96,7 \\
& Limítrofe & - & - \\
Colesterol & Alto & 2 & 3,3 \\
& Total & 61 & 100 \\
& Normal & 1 & 1,65 \\
& Limítrofe & 36 & 9,03 \\
Triglicerídeos & Alto & 24 & 9,4 \\
& Total & 61 & 100 \\
& Normal & 6 & 9,85 \\
& Limítrofe & 31 & 0,83 \\
& Alto & 24 & 9,4 \\
& Total & 61 & 100 \\
\hline & & &
\end{tabular}

Em relação à análise de correlação, pode-se observar que IM C, \% GC, CC e CQ apresentaram correlação significativa entre si ecom a pressão sistólica e diastólica. No entanto, não houve correlação significativa dessas variáveis com a glicose, colesterol etriglicerídeos (Tabela 5).

\section{Discussão}

0 estudo teve como população os idosos freqüentadores da UNATI da UNIFAL-M G, Alfenas, M G. Para Cervato et al. ${ }^{16}$, esses programas são estratégias para preparar a sociedade para uma realidade cada vez mais emergente: 0 aumento da população idosa no mundo, em especial nas nações em desenvolvimento. Este tipo de programa tem grande contribuição em manter as pessoas ativas e saudáveis, com maior autonomia, direito ao trabalho, ao lazer, à informação e à educação. No presente estudo, houve um predomínio da população feminina, constituindo $90,4 \%$ da amostra. Cervato et al. ${ }^{16}$ relataram elevada partici pação de mulheres em programas voltados para a terceira idade e a maior sensibilidade destas às necessidades demandadas para a promoção da saúde, em decorrência de suas experiências anteriores na utilização dos serviços de saúde, além da maior expectativa de vida para 0 sexo feminino.

Verificou-se que a maioria dos idosos apresentava bom nível socioeconômico, tanto de escolaridade quanto de renda familiar mensal. I sto pode ser devido ao fato de que os idosos com melhores rendas não necessitem complementar sua aposentadoria. E por apresentar maior escolaridade reconhecem a necessidade do autocuidado, que envolvemel horia na saúdee prática de atividades de lazer ${ }^{16}$.

$\mathrm{Na}$ análise das alterações sistêmicas decorrentes do envelhecimento relacionadas à idade, foi relatada plenitude pós-prandial, intestino preso, redução do apetite e alterações de olfato e paladar. Também se destacou o uso de prótese dentária e dor ao mastigar. Segundo Campos, M onteiro e Ornelas $^{17}$, as alterações na capacidade mastigatória do idoso são devidas ao aparecimento freqüente de cáries, próteses mau adaptadas e doenças periodontais. As alterações sensoriais, decorrentes das alterações fisiológicas do envelhecimento, podem estar associadas ao decréscimo do apetite.

0 uso de medicamentos entre os gerontes foi de $68,3 \%$. Passero e M oreira ${ }^{18}$ relataram que os idosos costumam utilizar muito mais medica- 
Tabela 4. Exames bioquímicos e pressão arterial segundo estado nutricional (IMC) dos idosos pertencentes à UNATI-UNIFAL-M G, Alfenas - M G, 2005.

\begin{tabular}{|c|c|c|c|c|c|c|c|c|}
\hline \multirow[t]{2}{*}{ Classificação } & \multicolumn{2}{|c|}{ Baixo peso } & \multicolumn{2}{|c|}{ Eutrofia } & \multicolumn{2}{|c|}{ Sobrepeso } & \multicolumn{2}{|c|}{ Total } \\
\hline & $\mathrm{n}$ & $\%$ & $\mathrm{n}$ & $\%$ & $\mathrm{n}$ & $\%$ & $\mathrm{n}$ & $\%$ \\
\hline \multicolumn{9}{|l|}{ Colesterol } \\
\hline Normal & ND & ND & 1 & 100,0 & ND & ND & 1 & 100 \\
\hline Limítrofe & 9 & 25,0 & 9 & 25,0 & 18 & 50,0 & 36 & 100 \\
\hline Alto & 2 & 8,3 & 8 & 33,3 & 14 & 58,3 & 24 & 100 \\
\hline \multicolumn{9}{|l|}{ Glicose } \\
\hline Normal & 10 & 16,9 & 18 & 30,5 & 31 & 52,2 & 59 & 100 \\
\hline Limítrofe & ND & ND & ND & ND & ND & ND & ND & ND \\
\hline Alto & 1 & 50,0 & ND & ND & 1 & 50,0 & 2 & 100 \\
\hline \multicolumn{9}{|l|}{ Triglicerídeos } \\
\hline Normal & ND & ND & 4 & 66,7 & 2 & 33,3 & 6 & 100 \\
\hline Limítrofe & 6 & 19,4 & 11 & 35,4 & 14 & 45,2 & 31 & 100 \\
\hline Alto & 5 & 20,8 & 3 & 12,5 & 16 & 66,7 & 24 & 100 \\
\hline \multicolumn{9}{|l|}{ Pressão arterial } \\
\hline Ideal & 8 & 33,3 & 6 & 25,0 & 10 & 41,7 & 24 & 100 \\
\hline Normal & 2 & 10,0 & 4 & 20,0 & 14 & 70 & 20 & 100 \\
\hline Normal * & 6 & 30,0 & 4 & 20,0 & 10 & 50 & 20 & 100 \\
\hline HAS I & 0 & 0 & 2 & 25,0 & 6 & 75,0 & 8 & 100 \\
\hline HAS II & 0 & 0 & 6 & 66,7 & 3 & 33,3 & 9 & 100 \\
\hline HAS III & ND & ND & ND & ND & ND & ND & ND & ND \\
\hline $\mathrm{HA}$ & 0 & 0 & 0 & 0 & 1 & 100 & 1 & 100 \\
\hline
\end{tabular}

ND: não detectado; *: normal alta; HAS: hipertensão arterial sistêmica; HA: hipertensão arterial.

Tabela 5. Correlação das variáveis antropométricas e pressão arterial dos idosos pertencentes à UNATIUNIFAL-MG, Alfenas - MG, 2005.

\begin{tabular}{lrrrrrr}
\hline & IMC & \multicolumn{1}{c}{$\%$ GC } & \multicolumn{1}{c}{ CC } & \multicolumn{1}{c}{ CQ } & \multicolumn{1}{c}{ PS } & \multicolumn{1}{c}{ PD } \\
\hline IMC & 1,00 & $0,705^{* *}$ & $0,841^{* *}$ & $0,899^{* *}$ & $0,364^{* *}$ & $0,276^{* *}$ \\
$\%$ GC & $0,705^{* *}$ & 1,00 & $0,666^{* *}$ & $0,723^{* *}$ & $0,317^{* *}$ & $0,305^{* *}$ \\
CC & $0,841^{* *}$ & $0,666^{* *}$ & 1,00 & $0,875^{* *}$ & $0,295^{* *}$ & $0,270^{*}$ \\
CQ & $0,899^{* *}$ & $0,723^{* *}$ & $0,875^{* *}$ & 1,00 & $0,306^{* *}$ & $0,242^{*}$ \\
PS & $0,364^{* *}$ & $0,305^{* *}$ & $0,295^{* *}$ & $0,306^{* *}$ & 1,00 & $0,884^{* *}$ \\
PD & $0,276^{* *}$ & $0,305^{* *}$ & $0,270^{*}$ & $0,242^{*}$ & $0,884^{* *}$ & 1,00 \\
\hline
\end{tabular}

*Correlação significante $(p<0,05)$;** Correlação significante $(p<0,01) \% G C$ : porcentagem de gordura corporal; $C C$ : circunferência da cintura; $C Q$ : circunferência do quadril, PS: pressão sistólica ePD: pressão diastólica

mentos do que pessoas de outra faixa etária; assim, estão mais propensos a sofrer seus efeitos adversos, incluindo as interações medicamentoalimento. Além disso, o fato de consumir mais medicamentos, concomitanteà menor eficiência da função orgânica, pode levar a um aumento do risco deintoxicação. A utilização, a longo prazo, de drogas terapêuticas que interferem na digestão, na absorção eno metabolismo de nutrien- tes pode, também, ocasionar perda de peso e conseqüente desnutrição e anorexia ${ }^{17}$.

Verificou-se nesse estudo maior prevalência de sobrepeso em relação à eutrofia e baixo peso. Estes resultados confrontam com estudos de Passero e M oreira ${ }^{18}$, que observaram maior prevalência de eutrofia $(36,7 \%)$ na população, seguido de sobrepeso $(33,3 \%)$ e de baixo peso (30,0\%). Esses resultados estão em concordân- 
cia com Santos e Sichieri ${ }^{19}$ e com o Relatório da OPAS ${ }^{20}$, no qual o sobrepeso teve sua prevalência aumentada na população brasileira, inclusiveem idosos, a partir de 1975, passando de 15,1 para $23,4 \%$ e de 22,9 para $32,4 \%$ para homens e muIheres idosas, respectivamente. Paral elamente a isso, o baixo peso ea eutrofia tiveram sua prevalência diminuída, o que demonstra a transição nutricional da população do país.

É importante que os valores de IM C se corre lacionem com outras medidas independentes da composição corporal, tendo em vista que o IM C não reflete a distribuição regional de gordura ocorrida com o processo de envelhecimento ${ }^{21}$. Em idosos, o emprego do IMC apresenta dificuldades em função do decréscimo da estatura, do acúmulo de tecido adiposo, da redução da massa corporal magra, da diminuição da quantidade de água do organismo e pela freqüente presença de patologias ${ }^{22}$. Assim, pela análise do $\%$ GC, constatou-se $62,2 \%$ de eutrofiae $37,8 \%$ de excesso de gordura corporal, o que pode ser explicado pelo declínio da massa magra, bem como do tecido ósseo e da água total do organismo, durante a fase posterior da vida adulta ${ }^{23}$.

No quediz respeito à hipertensão arterial sistêmica (HAS), a idade superior a 60 anos, em ambos os sexos, é fator de risco associado à patologia. No presente estudo, $46,3 \%$ dos idosos relataram possuir HAS, sendo que, de acordo com Taddei et al. ${ }^{24}$, a prevalência de HAS entre idosos foi de $65,0 \%$, podendo chegar atéa $80,0 \%$.

Considerando o IM C, 57,1\% dos indivíduos que possuíam pressão arterial elevada foram classificados com sobrepeso. Entretanto, 52,4\% dos indivíduos com pressão normal também apre sentaram sobrepeso. Esses achados se diferenciam dos trabal hos de Cabrera e Jacob Filho ${ }^{5}$, que encontraram entre idosos avaliados pelo $\mathrm{H}$ onolulu H eart Program uma relação direta entre IM C e HAS. Provavelmente, a não associação encontrada no presente trabal ho seja pela limitação do número de participantes.

A RCQ tem sido usada em estudos populacionais como preditora dos riscos de doenças cardiovasculares ${ }^{6}$. Os resultados de RCQ demonstraram-se elevados em $40,3 \%$ dos idosos.

Pela classificação da RCQ , observou-se 40,2\% dos idosos em risco alto e $12,2 \%$ em risco muito alto para desenvolver doenças cardiovasculares (DCV). Os gerontes com \% GC elevado apresentaram risco alto $(35,5 \%)$ e risco moderado $(38,7 \%)$ para DCV. Esses resultados sugerem uma alta predisposição desta população a desenvolver doenças crônicas não transmissíveis, visto que o acúmulo de gordura na região abdominal apresenta estreitas relações com doenças cardiovasculares. Entretanto, uma das principais limitações da RCQ éa ausência de pontos de corte específicos para a população idosa. U tilizamse, até o momento, os critérios propostos para os adultos jovens, sem considerar as alterações na distribuição de gordura inerentes ao processo deenvelhecimento ${ }^{25}$.

Observou-se que, dos 68 idosos que possuíam RCQ alto, 75\% apresentavam pressão arterial normal. Tal fato pode ser explicado pelo uso regular de anti-hipertensivos pelos idosos que já tinham conhecimento desua patologia eaos pontos de corte adotados para classificação de RCQ serem baseados em evidências de populações específicas de brancos, podendo, assim, não ser totalmente apropriados para idosos, mulheres e algumas etnias 5 . Q uando analisou-se os 21 indivíduos com HAS, 80,9\% apresentaram RCQ alto. Não foi observada associação entre a RCQ e a pressão arterial, assim como Ukoli et al. ${ }^{26}$, também, não verificaram relação entre os idosos nigerianos de ambos os sexos.

Outro fator quepode ter limitado o resultado foi o fato da aferição da pressão arterial ter sido realizada uma única vez, por setratar deum estudo transversal. De acordo com o National Institutes of $\mathrm{H}$ ealth ${ }^{27}$, o diagnóstico da H AS só é obtido pela referência do uso regular de medicamentos anti-hipertensivos ou duas medidas da pressão arterial (PA), com PA sistólica acima de 139 $\mathrm{mmHg}$ e/ou PA diastólica acima de $89 \mathrm{mmHg}$.

Segundo Passero e M oreira ${ }^{18}$, os indivíduos hipertensos, geralmente, têm aumento de peso corporal, associado a el evadas taxas de colesterol total e/ou triglicerídeos e glicemia. Lepira et al. ${ }^{28}$ evidenciaram que as médias de triglicerídeos e de colesterol total e suas frações estavam dentro da faixa de normalidade e foram similares em indivíduos com e sem hipertensão. Entretanto, 16\% dosindivíduos sem hipertensão e 23\% dos hipertensos estavam com elevados níveis de colesterol.

A associação da pressão sistólica com IM C também foi observada por El ayachi et al. ${ }^{29}$ epor Lepira et al. ${ }^{28} \mathrm{em}$ um estudo que mostrou que os hipertensos apresentaram maior IM C e CC.

A freqüência el evada dehiperglicemia (32,4\%) foi relatada por Pires, Gagliardi e Gorzoni ${ }^{30}$, em estudo retrospectivo, em indivíduos com idade igual ou superior a 60 anos. Este resultado difere do presente trabalho, no qual foram encontrados apenas 3,3\% de indivíduos com glicose sérica elevada.

Em relação aos níveis séricos de colesterol, 
$39,4 \%$ da amostra apresentavam valor elevado Freqüência próxima $(30,0 \%)$ foi encontrada por Taddei et al. ${ }^{24} \mathrm{em}$ um estudo multicêntrico com idosos maiores de 65 anos, cuja população total era de 2.196 indivíduos.

No presente estudo, observou-se que 50,8 e $39,4 \%$ dos gerontes apresentaram valores de triglicerídeos limítrofes e elevados, respectivamente. Esses resultados foram superiores aos encontrados por Pires, Gagliardi e Gorzoni ${ }^{30}$, cuja pre valência foi de $20,0 \%$ de idosos com triglicerídeos alterado. Segundo Duarte e Nascimento ${ }^{31}$ e Amado e Arruda ${ }^{32}$, os níveis séricos de triglicerídeos tendem a se elevar com a idade, refletindo a incapacidade do idoso de controlar tais níveis.

Estudo realizado com mulheres idosas pelo Nurse's H ealth Study demonstrou que as obesas apresentavam 39 vezes mais chances de desenvolver diabetes que as eutróficas ${ }^{33}$. Entretanto, os dados encontrados no presente estudo não demonstraram associações relevantes entre sobrepeso eglicose sérica elevada, já que, dos indivíduos que apresentaram sobrepeso, somente 3,3\% possuíam glicose elevada.

Quanto ao colesterol sérico, ficou evidenciado que $50 \%$ dos indivíduos com níveis limítrofes estavam com sobrepeso. E que 58,3\% com níveis elevados apresentaram sobrepeso. Entretanto, não foi encontrada associação entre colesterol e sobrepeso. Cercato et al. ${ }^{33}$, em estudo com 1.213 indivíduos, também não encontraram associação entre níveis elevados de colesterol e obesidade. Em relação ao triglicerídeos, observou-seque a metade dos indivíduos classificados com sobrepeso $(50,0 \%)$ teve níveis elevados. Esses resultados corroboram e até mesmo apresentam maiores prevalências quando comparados com os de N jelekela et al. ${ }^{34}$, que demonstraram entre mulheres obesas maiores níveis de triglicerídeos, colesterol total e LDL. E ainda com os achados deEl ayachi et al. ${ }^{29}$, que observaram níveis eleva- dos de triglicerídeos (11,8\%) e colesterol (10\%) entre mulheres marroquinas.

Apesar de El ayachi et al. ${ }^{29}$ terem observado que triglicerídeos se correlacionaram positivamenteesignificativamentecom IM C eainda $\mathrm{Nje}$ lekela et al. ${ }^{34}$ terem encontrado correlação positiva do IMC e CC com colesterol, triglicerídeos para ambos os sexos, as correlações entre IM C com os dados bioquímicos estudados no presente trabalho estão de acordo com os achados de Baral et al..$^{35}$, que encontraram, em idosos no Nepal, correlação positiva do IM C com triglicerídeos, colesterol e frações, embora sem significado estatístico.

\section{Conclusão}

A população estudada era composta na sua maioria por mulheres, com bom nível de escolaridade e renda familiar. Os idosos pertencentes à UNA$\mathrm{TI}$ apresentaram risco muito alto para desenvolvimento de doenças crônicas não transmissíveis. Observaram-se também altas prevalências de sobrepeso e excesso de GC, colesterol etriglicerídeos elevados, além de hipertensão arterial.

Portanto, a avaliação nutricional antropomé trica e bioquímica do idoso são de extrema importância para a identificação das alterações que acompanham o envelhecimento ese refletem no estado nutricional e no possível desenvolvimento de doenças.

Conclui-se, diante destes resultados, que as atividades desenvolvidas em programas abertos para a terceira idade devem ser voltadas para as necessidades e expectativas da população, promovendo melhora da qualidade de vida para os idosos. Programas de educação nutricional continuada e de monitoramento do estado nutricional e de saúde são necessários para melhoria da qualidade de vida destes indivíduos. 


\section{Colaboradores}

RR Silva coordenou esupervisionou todas as etapas executadas no projeto, orientou a parte me todológica e intelectual do trabalho, além de auxiliar na redação final do manuscrito; HSD M artino co-orientou todas as etapas executadas no projeto e auxiliou na interpretação dos dados, redação intelectual e final do manuscrito; JM Bueno auxiliou na redação do projeto e executou as etapas de levantamento dos dados e redação do manuscrito; M FS Fernandes executou as etapas de levantamento dos dados e participou da redação inicial do manuscrito e LS Costa executou as etapas de levantamento dos dados e participou da redação inicial do manuscrito.

\section{Referências}

1. M athias TAF, Jorge MHPM, Laurenti R. Doenças cardiovasculares na população idosa. Análise do comportamento da mortalidade em município da região Sul do Brasil no período de 1979 a 1998. Arq. Bras. Cardiologia 2004; 82(6):533-541.

2. Da Cruz IBM, Almeida M SC, Schwanke CHA, M origuchi $\mathrm{EH}$. Prevalência de obesidade em idosos longevos e sua associação com fatores de risco de morbidades cardiovasculares. Rev. Assoc. Med. Bras 2004; 50(2):172-177.

3. Menezes TN, Marucci MFN. Antropometria de idosos residentes em instituições geriátricas, Fortaleza, CE. Rev. Saúde Pública 2005; 39(2):169-175.

4. Silva R, Rauen MS, M oreira EAM. Hábitos alimentares em idosos. Rev. Bras. Nut. Clín. 2005; 20(4):246250.

5. Cabrera MAS, Jacob Filho WJ. Obesidade em idosos: prevalência, distribuição e associação com hábitos e co-morbidades. Arq. Bras. Endocrinol. Metab. 2001; 45(5):494-501.

6. Cabrera MAS, Gebara OCE, Diament J, Nussbacher A, Rosano G. M etabolic syndrome abdominal obesity, and cardiovascular risk in elderly women. Int J Cardiol 2007; 114:224-229.

7. Durnin JVGA. Anthropometrics methods of assessing nutritional status. In: Horwits A, DM M acFadyen, Munro H, Scrimshaw NS, Steen B, TF Williams, editors. Nutritional in the elderly. Oxford: Oxford University Press; 1989. p. 15-32.

8. Jelliffe DB. Evolución del estado de nutrición de la comunidad. Ginebra: Organización Mundial de la Salud; 1968.

9. Nutrition Screening Initiative: Nutrition Screening. NSI. M anual for Professionals Caring for Older Americans. Washington, D.C: Nutrition Screening Initiative; 1991.

10. Bray GA, Gray DS. Anthropometric measurements in the obese. In: Lohman TG, Roche AF, Martorelli R, editors. Anthropometric standardization reference manual. Champaign: Human Kinetics; 1988. p. 131-136.

11. Coelho AK, Fausto MA. Avaliação do estado nutricional. In: Marciel A, organizador. Avaliação em geriatria. Rio de Janeiro: Revinter; 2002.

12. Lip GY, Chung N. Hypertension patients at risk. London: Servier; 2002.

13. Sociedade Brasileira de Cardiologia. [acessado 2005 Fev 25]. Disponível em: http://www.cardiol.br.

14. Dean AG, Dean JA, Coulombier D, Brendel KA, Smith DC, Burton AH, Dickers RC. Epi Info, Version 6:04b: a word processing database and statistics program for public health on IBM-compatible microcomputers [programa de computador]. AtIanta, Georgia: Centers for Disease Control and Prevention; 1996.

15. Statistical Package for the Social Science for Windows. SPSS/Pc version 10.0 [programa de computador]. Chicago: SPSS; 2000.

16. Cervato AM, Derntl AM, Latorre MRDO M arucci, MFN. Educação nutricional para adultos e idosos: uma experiência positiva em U niversidade Aberta para a Terceira Idade. Rev. Nutrição 2005; 18(1):41-52. 
17. Campos MTFS, M onteiro JBR, Ornelas APRC. Fatores que afetam o consumo alimentar e a nutrição do idoso. Rev. Nutrição 2000; 13(3):157-165.

18. Passero V, Moreira EAM. Estado nutricional de idosos e sua relação com a qualidade de vida. Rev. Bras. Nut. Clín. 2003; 18(1):1-7.

19. Santos DM, Sichieri R. Índice de massa corporal e indicadores antropométricos de adiposidade em idosos. Rev. Saúde Pública 2005; 39(2):163-168.

20. Organização Pan-Americana da Saúde. Ano internacional das pessoas idosas. Envelhecimento. Mitos na Berlinda. Brasília: OPAS; 1999.

21. Anjos LA. Índice de massa corporal (massa corporal. Estatura - 2) como indicador do estado nutricional de adultos: revisão da literatura. Rev. Saúde Pública 1992; 26(6):431-436.

22. Bedogni G, Pietrobelli A, Heymsfield SB, Borghi A, Manzieri AM, Morini P, Battistini N, Salvioli G. Is body mass index a measure of adiposity in elderly women? Obes. Res 2001; 9(1):17-20.

23. Cordeiro RG, Moreira EAM . Avaliação nutricional subjetiva global do idoso hospitalizado. Rev. Bras. Nut. Clín. 2003; 18(3):106-112.

24. Taddei CFG, Ramos LR, Moraes JC. Estudo multicêntrico de idosos atendidos em ambulatórios de cardiologia e geriatria de instituições brasileiras. Arq. Bras. Cardiol. 1997; 69(5):327-333.

25. Sampaio LR. Avaliação nutricional e envelhecimento. Rev. Nutrição 2004; 17(4):507-514.

26. Ukoli FA, Bunker $\mathrm{CH}$, Fabio A, Olomu AB, Egbagbe $E E$, Kuller LH. Body fat distribution and other anthropometric blood pressure correlates in a $\mathrm{Ni}$ gerian urban elderly population. Cent. J. M ed. 1995; 41(5):54-161.

27. Joint National Committee. Prevenção, detecção, avaliação e tratamento da pressão arterial elevada. Bethesda: National Institute of $\mathrm{H}$ ealth; 1997.

28. Lepira FB, M 'Buyamba-Kabangu JR, Kayembe KP, $N$ seka M N. Correlates of serum lipids and lipoproteins in Congolese patients with arterial hypertension. Cardiovasc J S Afr. 2005; 16(5):249-255.
29. El ayachi M, M ziwira M, Vincent S, Defoort C, Portugal H, Lairon D, Belahsen R. Lipoprotein profile and prevalence of cardiovascular risk factors in urban Moroccan women. Eur J Clin Nutr. 2005; 59(12):1379-1386.

30. Pires SL, Gagliardi RJ, Gorzoni ML. Estudo das freqüências dos principais fatores de risco para acidente vascular cerebral isquêmico em idosos. Arq. Neuro-Psiquiatr. 2004; 62(3b): 844-851.

31. Duarte ALN, Nascimento ML. Condutas dietéticas. In: Netto MP, organizador. Gerontologia - a velhice em visão globalizada. São Paulo: Atheneu; 2000. p. 262-272.

32. Amado TCF, Arruda IKG. Hipertensão arterial no idoso e fatores de risco associados. Rev. Bras. Nut. Clín. 2004; 19(2):94-99.

33. Cercato C, M ancini MC, Arguello AM, Passos VQ, Villares SM, Halpern A. Systemic hypertension, diabetes mellitus, and dyslipidemia in relation to body mass index: evaluation of Brazilian population. Rev. Hosp. Clín. Fac. M ed. S. Paulo 2004; 59(3):113-118.

34. Njelekela MA, Negishi $H, N$ ara $Y$, Sato $T$, Tomohiro M, Kuga S, Noguchi T, Kanda T, Yamori M, Mashalla Y, Liu LJ, I keda K, M tabaji J, Yamori Y. O besity and lipid profiles in middle aged men and women in Tanzania. East Afr M ed J. 2002; 79(2):58-64.

35. Baral N, Jha P, Sridhar MG, Karki P, Sharma SK, Khambu B.Association of lipid profile and body mass index (BMI) in hypertensive patients of eastern Nepal. JNMA J Nepal Med Assoc. 2006; 45(163):306-309.

Artigo apresentado em 25/04/2007

Aprovado em 06/06/2007 